\title{
A ZigBee multipath routing algorithm based on ant colony algorithm
}

\author{
Chaohua $\mathrm{Wu}^{1}{ }^{1}$,, Yunfei $\mathrm{Li}^{1}$ and Juncheng $\mathrm{Jia}^{1}$ \\ ${ }^{1}$ School of Soochow University, Suzhou 215000, China; \\ att15956001264@163.com
}

Keywords: ant colony algorithm, multipath routing, AODVjr, AOMDV

\begin{abstract}
There are many problem in moving process of wirless sensor network such as packet loss and link failures. This paper proposed a ZigBee multipath routing protocol Ant-AOMDVjr based on ant colony algorithm after the deep research of ant colony algorithm,AODVjr algorithm and AOMDV routing algorithm. Ant-AOMDVjr combines the advantages of ant colony algorithm,AODVjr and AOMDV,considers the link quality, routing hops and node energy into the router discovery and router maintenance. Simulation based on NS2 software indicates that the packet delay and end-to-end transmission delay is improved compared with AODVjr and AOMDV algorithm.
\end{abstract}

\section{Introduction}

Zigbee technology ${ }^{[1]}$ is a low-rate wireless connectivity technology for fixed or mobile devices, with very low complexity,cost and power,widely used in wireless sensor networks. ZigBee protocol stack is built on the IEEE 802.15.4 standard,it specifies the standards for the network layer, application layer, and security service layer.It supports star network,mesh topology and tree topology. The star network has a simple structure,the communication distance between the nodes is shorter and the network function is less scalable; The mesh structure has strong robustness, can provide multiple data channels, is a highly reliable detection network; The tree network combines the advantages of star networks and mesh networks, with good scalability.

ZigBee network supports AODVjr algorithm ${ }^{[2]}$ and Cluster-Tree algorithm ${ }^{[3]}$, in which AODVjr algorithm is a simplified version of AODV algorithm, mainly used in mesh network; Cluster-Tree algorithm is mainly used for cluster tree network.In the literature 4 , the problem of Cluster-Tree ${ }^{[4]}$ algorithm can not provide the best routing path in general. A distributed shortcut tree algorithm STR is proposed, which can provide a better routing path based on only a limited adjacency table maintenance overhead and reduce the transmission delay.

ZigBee network in the process of moving and harsh environments will encounter serious packet loss and link failure problems, especially when the ZigBee network nodes in the larger number these problems will be more obvious. In this paper, the ant colony algorithm is applied to the AODVjr algorithm, and combined with the AOMDV algorithm. A ZigBee multipath routing algorithm based on ant colony algorithm is proposed and simulated compared with AOMDV and AODVjr algorithm by simulation software.

\section{Relevant definition}

\subsection{Ant Colony Algorithm}

The ant colony algorithm ${ }^{[5]}$ is composed of $\mathrm{M}$. Dorigo and other scholars put forward oneOptimization Algorithm for Simulation of Ant Colony Behavior in Nature, practice has proved that it can solve many combinatorial optimization problems. Ant colony algorithm has the characteristics of positive feedback, parallelism and greedy heuristic search, which makes it possible to apply the topology change of mobile network to the effect of routing algorithm. 
The classic Symmetry TSP problem ${ }^{[6]}$ can be solved by the ant colony algorithm, which is used to describe the optimized ant colony algorithm:

Set $\operatorname{ants}(m)$ in accordance with a certain initialization rules are distributed in citys $(n)$, these citys collection are $C=\left\{c_{1}, c_{2}, \cdots, c_{n}\right\}$, ants $k(k=1,2, \cdots, m)$ in the movement, the ants will be based on the path of the number of pheromone and path of the heuristic information to calculate the probability of transfer, $p_{i, j}^{k}(t)$ is used to indicate the probability of ants moving from a city to another:

$$
p_{i, j}^{k}(t)=\left\{\begin{array}{l}
\frac{\left[\tau_{i, j}(t)\right]^{\alpha}\left[\eta_{i, j}(t)\right]^{\beta}}{\sum\left[\tau_{i, s}(t)\right]^{\alpha}\left[\eta_{i, s}(t)\right]^{\beta}}, \\
j \in \text { allowed }_{k}, s \subset \text { allowed }_{k} \\
0, \quad \text { otherwise }
\end{array}\right.
$$

In Eq. (1), $\tau_{i, j}(t)$ is the number of pheromones on path $(i, j)$ at time $t$, and the value at the initial moment is $\tau_{i, j}(0)=$ const, allowed $k=\left\{C-\operatorname{tabu}_{k}\right\}$, Where $\operatorname{tabu}_{k}(k=1,2, \cdots, m)$ is the city where the ant $k$ has traveled, and $\eta_{i, j}(t)$ is the degree to which the ants are moved from city $i$ to $j$ :

$$
\eta_{i, j}(t)=1 / d_{i j}
$$

In Eq. (2), $d_{i j}$ is the distance from city $i$ to $j$.

The ant will update the pheromone quantity after one step or complete the traversal of all the cities, and the pheromone number on the path $(i, j)$ at time $(t+n)$ is updated according to Equation $(3),(4)$.

$$
\begin{gathered}
\tau_{i, j}(t+n)=(1-\rho) \tau_{i, j}(t)+\Delta \tau_{i, j}(t) \\
\Delta \tau_{i, j}(t)=\sum_{k-1}^{m} \Delta \tau_{i, j}^{k}(t)
\end{gathered}
$$

In Eq. (3) and(4), $\rho$ is the volatilization coefficient of pheromone, which among $[0,1], \Delta \tau_{i, j}(t)$ is the pheromone increment on this cycle path $(i, j), \Delta \tau_{i, j}^{k}(t)$ is the number of pheromone that the ant $k$ remains on path $(i, j)$ in this cycle.

\subsection{The AODVjr algorithm}

AODVjr algorithm is a simplified version of $\mathrm{AODV}^{[7]}$, taking into account the cost, energy efficiency, ease of use and other factors, the AODV made the following changes:

AODVjr algorithm cancel the node serial number and prohibit the middle node to reply to RREP, stipulate only the target node can reply to RREP, effectively ensure the route without loop; AODVjr algorithm cancel the HELLO message, the target node send regular KEEP ALIVE connection information to the source node to maintain the route, it does not require additional overhead for routing maintenance if the communication is bi-directional;The AODVjr algorithm uses local remediation for link interrupts. If the repair fails, the RERR is sent to the source node of the packet, and the RERR format is simplified to include only one unreachable node.

Compared with the AODV algorithm, the AODVjr algorithm has a lot of improvements, but its source node only maintains one route to the destination node, can not adapt to the ZigBee network with frequent topology changes and more interference in the communication environment. When the route fails, Routing discovery, resulting in increased network latency and other issues, resulting in network performance degradation.

\subsection{The AOMDV algorithm}

The AOMDV algorithm is an extension of the AODV algorithm, and there are many similarities between the AODV algorithm, which are based on the distance vector concept and using the hop-by-hop method. The idea of the protocol is to establish multiple link disconnection routes in routing discovery. And select one of them as the primary route,other as the standby route. Enable the 
alternate route when the primary route fails. Re-route the route only if all routes fails. Thus reducing the number of route discovery, reduce end-to-end delay and improve network performance.

The AOMDV algorithm discovers many multiple routes are independent of each other in route discovery, so that the route can be switched to the standby route when the primary route fails, and there is no need to reroute the route. However, the AOMDV algorithm does not maintain the standby route, which can cause the standby route fails when the network topology changed or the communication environment interference more.

\section{Ant-AOMDVjr}

Ant colony algorithm using distributed parallel computing mechanism, easy to combine with other methods, with strong robustness. The application of ant colony algorithm to ZigBee network is helpful to speed up route discovery and alleviate the situation of network congestion.Combining the the advantages of AOMDV algorithm and AODVjr algorithm, the ZigBee multipath routing algorithm Ant-AOMDVjr based on ant colony algorithm is proposed.

Ant-AOMDVjr is based on the AOMDV algorithm, the ant colony algorithm is applied to the routing discovery. The "pheromone" strength, the number of hops, the link quality and the node energy are the routing discovery criteria,the link communication quality and the node energy are perceived during route maintenance. When the communication quality of the link or node energy reaches the alert value for the first time, it is ready to switch the link. When the second time the alarm value is reached, the route is switched, only when all the backup route failure Re-route discovery.

\subsection{The algorithm design}

Ant-AOMDVjr algorithm abstracts ants into forward ant and backward ants in routing discovery, corresponding to RREQ messages and RREP messages in AODVjr algorithm. The algorithm of the routing table structure shown in Fig. 1, in the AOMDV routing table on the basis of the increase:

1) Pheromone: Used to reflect the changes in pheromones and the calculation of the selection probability.

2) Link quality: This algorithm uses LQI (Link Quality Indicator) ${ }^{[8]}$ link quality indicator as the basis for measuring the quality of the link. LQI can be obtained directly from the structure after receiving the data. If the structure after the data is received is rdp, LQI $=$ rdp-> LinkQuality. LQI has a range of values in the determined network device, and its value is proportional to the link quality. The weights of the link quality between two adjacent nodes are as follows:

$$
P S_{i, j}=\frac{L Q I_{i, j}-L Q I_{\min }}{L Q I_{\max }-L Q I_{\min }}
$$

$P S_{i, j}$ is the link weight between nodes $i$ and $j$ and proportional to the link quality; $L Q I_{i j}$ is the LQI value sent by node $i$ to node $j ; L Q I_{\max }$ and $L Q I_{\min }$ are the upper and lower limits of LQI.

The link quality normalization value of the source node to the destination node is calculated as follows:

$$
P S=\prod_{i, j \in \text { Path }} P S_{i, j}
$$

3) Node energy: the battery value of the node.

4) The select probability: The next hop node routing probability, its value is calculated as follows:

$$
p_{i, j}^{k}(t)=\left\{\begin{array}{l}
\frac{\left[\tau_{i, j}(t)\right]^{\alpha}\left[\eta_{i, j}(t)\right]^{\beta}\left[P S_{i, j}(t)\right]^{\gamma}\left[\xi_{i, j}(t)\right]^{\delta}}{\sum\left[\tau_{i, s}(t)\right]^{\alpha}\left[\eta_{i, s}(t)\right]^{\beta}\left[P S_{i, s}(t)\right]^{\gamma}\left[\xi_{i, s}(t)\right]^{\delta}}, j \in \text { allowed }_{k}, s \subset \text { allowed }_{k} ; \\
0, \quad \text { otherwise }
\end{array}\right.
$$


In Eq.(7), $p_{i, j}^{k}(t)$ is the probability that the $k$ th control message is transferred from node $i$ to node $j$ at time $t ; \tau_{i, j}(t)$ denotes the number of pheromones on the path $(i, j)$ at time $t ; \eta_{i, j}(t)$ denotes the heuristic function of node $i$ to node $j$ at time $t$, that is, the reciprocal of the number of hops of the source node to the current node; $P S_{i, j}(t)$ is the weight of the link quality of node $i$ to node $j$ at time $t ; \xi_{i, j}(t)$ is the energy value of node $j$ at $t$; allowed als $_{k}$ is set of neighbor nodes of node i. $\alpha, \beta, \gamma, \delta$ are the pheromone energetic factors, the number of heuristic factors, the link quality heuristic factor and the node energy heuristic factor respectively. The relative pheromone, hops, link quality and node energy are described in the state transition importance.

\begin{tabular}{|c|c|c|c|c|c|c|c|c|}
\hline \multirow{4}{*}{ 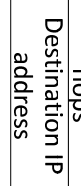 } & \multirow{4}{*}{ ס } & \multicolumn{7}{|c|}{ Path list } \\
\hline & & Next hop 1 & Hop count 1 & Pheromone 1 & Select probability 1 & Last jump 1 & Expiry time 1 & Link quality 1 \\
\hline & & Next hop 2 & Hop count 2 & Pheromone 2 & Select probability 2 & Last jump 2 & Expiry time 2 & Link quality 2 \\
\hline & & $\ldots$ & $\ldots$ & $\ldots$ & $\ldots$ & $\ldots$ & $\ldots$ & $\ldots$ \\
\hline
\end{tabular}

Fig. 1 The algorithm routing table structure

The pheromones on the path are updated according to Eqs. (3) and (4), and the pheromones on which the packets remain on the path are calculated according to Eq. (8)

$$
\Delta \tau_{i, j}^{k}(t)=Q \cdot \Phi_{i, j}
$$

In the Eq.(8), $Q$ is the pheromone intensity, and $\Phi_{i, j}$ is the degree of congestion of the network link. The value is the ratio of the total queue in the remaining space of the MAC layer interface cache queue.

\subsection{The Route discovery}

The routing of the algorithm is shown in Fig. 2. This algorithm eliminates the node serial number, disables the intermediate node from responding to RREP, and adds the link quality field PS to the RREP and RREQ messages.

When the source node needs to send data to a destination node, the source node will first see whether it has valid routing information to reach the destination node in its routing table. If so, select the path to send the route. If there is no source node, broadcast the RREP Message, according to the Eq.(7) to select the largest probability of the neighbor node as a next hop, to establish a path; then select the next high probability of the neighbor node as the next hop to establish an alternate path. When receiving the RREQ message, the intermediate node calculates the link quality $P S_{i, j}$ of the preceding node to the node according to Eq. (5), multiplies the link quality field PS in the RREQ message, and replaces the calculated value with the original link quality Field, and then forward; After receiving the RREQ request, the intermediate node directly rejects the RREQ request, the destination node only answers the routing requests from the neighbor nodes of different source node. After the RREP packet is generated, the reverse route established along the RREQ in the form of unicast is propagated to the source node and the routing table is updated according to the update rule of the pheromone.

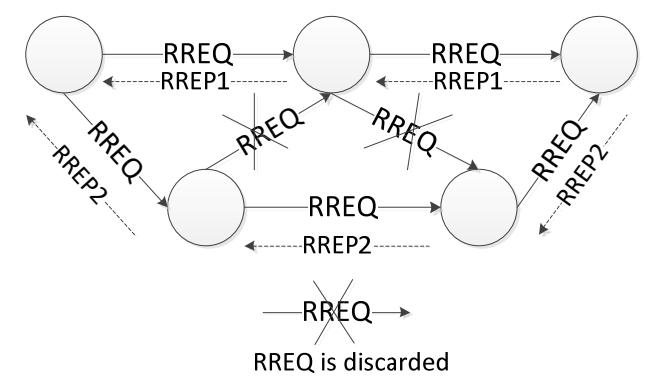

Fig. 2 This algorithm routing discovery process 


\subsection{The Routing maintenance}

Ant-AOMDVjr algorithm uses the AODVjr algorithm KEEP ALIVE way to carry out route maintenance. The destination node periodically sends KEEP ALIVE information to the source node, which contains link quality and node energy. This method is comparable to the AODV HELLO message broadcast, which can reduce the overhead of the network. When the link quality or node energy drops to the alarm value for the first time, the source node sends information to each standby link to get the latest link quality and node energy; The failed path for a change in the network topology is removed from the routing table; When the link quality or node energy is reduced to the alarm value for the second time, the path is switched, that is, the path of the maximum selection probability is selected from the standby link as the main path, and the original path is removed from the routing table path; Reroute discovery when no path is available.

\section{Experiment}

In this paper, the algorithm is simulated in NS2 simulation environment, and compared with AODVjr algorithm and AOMDV algorithm in the same network environment. In the simulation environment, 60 nodes are moved, the node moves in any direction, the maximum speed is $30 \mathrm{~m} / \mathrm{s}$, the movement range is $1000 \mathrm{~m} \times 1000 \mathrm{~m}$ square area, the simulation time is $300 \mathrm{~s}$, send $0 \sim 3 \mathrm{CBR}$ packets per second and the size of each CBR packet is 512 bytes. In this algorithm, $Q$ is 0.35 , and $\alpha, \beta, \gamma, \delta$ are 2. This simulation is aimed at the packet delivery rate and the end-to-end average delay of the three routing protocols when the nodes move.

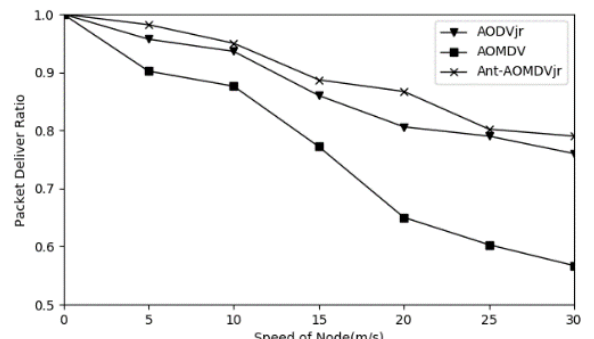

Fig. 3 Group delivery rate

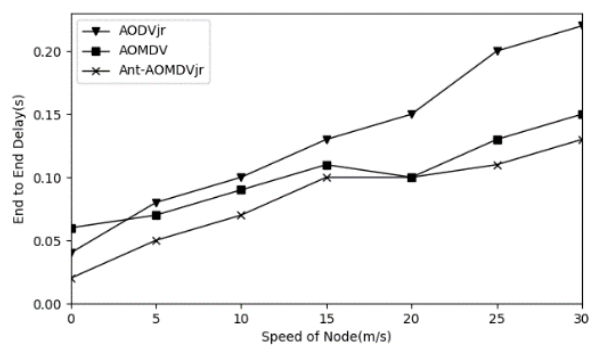

Fig. 4 End-to-end delay

It can be seen from Fig. 3, the three road protocol packet delivery rate showed a decreasing trend with the increase in the speed of movement. When the speed of the node increases, the probability of the link disconnection becomes larger for AODVjr only found one path in route discovery, resulting in an increase in the number of route reorganizations and the overhead of the network. The AOMDV algorithm has an alternate path, but does not maintain its backup path, resulting in the standby path may have failed when switching to an alternate path; In this paper, we maintain the path of multiple nodes and choose the route with the strongest comprehensive ability to communicate as the main route, and can switch to the standby path in time, thus increasing the delivery rate of the packet.

Fig. 4 shows that the end-to-end average delay of the three routing protocols increases with the mobility of the nodes. Since the AODVjr algorithm only selects the shortest path, it can not sense the delay and the network load, and it is prone to congestion. Since the AOMDV algorithm also selects the routing hop count as the criterion of routing and does not maintain the standby link, the average end-to-end delay of the AOMDV algorithm is also slightly higher. Ant-AOMDVjr algorithm combines the link quality, node energy, hop count and other information, can choose a better route, reducing the end to end of the delay.

\section{Summary}

Based on the ant colony algorithm, AODVjr and AOMDV routing protocol are analyzed in detail, a ZigBee multipath routing algorithm based on ant colony algorithm is proposed. Ant-AOMDVjr algorithm considers the number of hops, link quality, node energy, node congestion and other factors. Multiple paths are found in route discovery, after the link quality of the main route or the node energy 
drops to the alert value, the source node can be informed of the routing timely. The simulation results show that the AODVjr and AOMDV routing protocols have higher packet delivery rate and lower end-to-end delay, which is better in the network environment of node movement.

\section{References}

[1] ZigBee Document 053474r06. Version 1.0. ZigBee Specification [S]. ZigBee Alliance, 2004.

[2] Chakeres I D, Klein-Berndt.AODVjr, AODV simplified [J].Mobile Computing and Communication Review, 2002, 6(3):100-101.

[3] Kim T, Kim D, Park N,et al.Shortcut tree routing in ZigBee network[EB/OL].[2008-02-16]. http://resl.icu.ac.kr/ damiano/proc/iswpc2007_1.pdf.

[4] Kin T,Kim S H,Yang J,et al.Neighbor table based shortcut tree routing in ZigBee wirless networks[J].IEEE Transactions on Parallel and Distributed Systems,2014,25(3):706-716

[5] Colorni A, Dorigo M, Maniezzo V, et al. Distributed optimization by ant colonies [A] . Procof European Conf on Artificial Life[C].Paris, 1991.134-142.

[6] Dorigo M, Maniezzo V, Colorni A. Ant system: Optimization by a colony of cooperating Agents [J] . IEEE Transon Systems, Man, and Cybernetics - Part B,1996, 26(1) : 29-41.

[7] Perkins C E,Royer E M. Ad-Hoc on-demand distance vector routing(AODV)[R].California:University of California,2013

[8] REIJERS N, HALKES G, LANGENDOEN K. Link layer measurements in sensor networks[A].Proceedings of First International Conference on Mobile Ad Hoc and Sensor Systems [C]. Fort Lauderdale,USA, 2004. 224-234. 